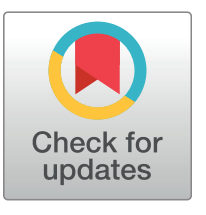

CORRECTION

\title{
Correction: Genetic interactions among ADAMTS metalloproteases and basement membrane molecules in cell migration in Caenorhabditis elegans
}

\author{
Ayaka Imanishi, Yuma Aoki, Masaki Kakehi, Shunsuke Mori, Tomomi Takano, \\ Yukihiko Kubota, Hon-Song Kim, Yukimasa Shibata, Kiyoji Nishiwaki
}

\section{Notice of Republication}

This article was republished on January 25, 2021, to correct errors in the symbols. In the originally published version, the Greek "alpha" symbol $(\alpha)$ appeared incorrectly as either the letter "a" or as a corrupted symbol. Please download this article again to view the correct version. The originally published, uncorrected article and the republished, corrected articles are provided here for reference.

\section{Supporting information}

S1 File. Originally published, uncorrected article.

S2 File. Republished, corrected article.

\section{Reference}

1. Imanishi A, Aoki Y, Kakehi M, Mori S, Takano T, Kubota Y, et al. (2020) Genetic interactions among ADAMTS metalloproteases and basement membrane molecules in cell migration in Caenorhabditis elegans. PLoS ONE 15(12): e0240571. https://doi.org/10.1371/journal.pone.0240571 PMID: 33264296

G

\section{OPEN ACCESS}

Citation: Imanishi A, Aoki Y, Kakehi M, Mori S, Takano T, Kubota Y, et al. (2021) Correction: Genetic interactions among ADAMTS metalloproteases and basement membrane molecules in cell migration in Caenorhabditis elegans. PLoS ONE 16(12): e0261880. https://doi. org/10.1371/journal.pone.0261880

Published: December 21, 2021

Copyright: @ 2021 Imanishi et al. This is an open access article distributed under the terms of the Creative Commons Attribution License, which permits unrestricted use, distribution, and reproduction in any medium, provided the original author and source are credited. 